\title{
Variables psicosociales del entorno comunitario asociadas a procesos de desadaptación social en adolescentes: reflexiones a partir de un estudio de caso*
}

\author{
Psychosocial Variables of Community Environment Linked to Social \\ Maladjustment Processes in Adolescents: Reflections from a Case Study
}

Recibido: junio 3 de 2012 | Revisado: agosto 5 de 2012 | Aceptado: septiembre 20 de 2012

\author{
Alba Zambrano CONSTANZO ** \\ JAIME MUÑOZ VIDAL *** \\ MARCO GONZÁLEZ SÁNCHEZ ***** \\ Universidad de La Frontera, Temuco, Chile
}

\section{RESUMEN}

Se analiza la incidencia de un conjunto de variables relacionales y del entorno social construido que actúan como factores de riesgo en procesos de desadaptación social de adolescentes en un barrio de Temuco, Chile. Como técnicas para la producción de datos, se emplearon: la observación participante, las entrevistas focalizadas, el análisis documental, los talleres de discusión grupal y el análisis estructural de redes. En un análisis en progreso de los datos, bajo la lógica de construcción de teoría desde la base, se caracteriza la dinámica barrial en cinco dimensiones: convivencia comunitaria, acción institucional en la comunidad, norma comunitaria, abordaje familiar del comportamiento infantil y adolescente y apropiación del espacio público. Se propone que estas dimensiones en el polo negativo actuarían como variables criminógenas en el espacio comunitario.

Para citar este artículo. Zambrano, A., Muñoz, J. \& González, M. (2012). Variables psicosociales del entorno comunitario asociadas a procesos de desadaptación social en adolescentes: reflexiones a partir de un estudio de caso. Universitas Psychologica, 11(4), 1135-1145

Queremos agradecer a los habitantes del barrio Vista Verde por su colaboración en la realización de este trabajo, el cual no podría haberse realizado sin su participación.

*** Docente del Departamento de Psicología y Directora Alterna Proyecto FONDEF D08I1205 titulado "Estrategia ecosistémica especializada de intervención diferenciada para favorecer la integración psicosocial de adolescentes infractores de ley". Email: albaz@ufro.cl. ResearcherID: Zambrano, A. G-9067-2012.

**** Psicólogo, estudiante de Magíster y Especialización en Psicología Comunitaria, encargado de terreno, subcomponente Fortalecimiento Comunitario, Proyecto FONDEF D08I1205.E-mail: jmunozvidal@ gmail.com. ResearcherID: Muñoz, J. G-8847-2012.

****** Psicólogo, investigador de terreno, Proyecto FON. DEF D08I1205. E-Mail: magsallanico@gmail.com. ResearcherID: Gonzalez, M. G-9220-2012.

Palabras clave autores

Adolescencia, proceso de desadaptación social, entorno comunitario, factores psicosociales, variables comunitarias criminógenas.

Palabras clave descriptores

Psicología Social-Comunitaria, espacio público-comunitario, investigación cualitativa.

\footnotetext{
A B S T R A C T

This article analyses the incidence of a group of relational and social context constructed variables that act as risk factors in processes of social maladjustment of adolescents in a neighborhood belonging to Temuco city, Chile. The techniques used to produce the data were participant observation, focalized interviews, document analysis, group discussion workshops and structural analysis of networks. In a progressive analysis of the data, using grounded theory, the neighborhood dynamics is characterized by five dimensions: community cohabitation, institutional action in the community, community norm, family approach to child and adolescent behavior; and community taking of the public space. It is proposed that these dimensions in its negative pole would act, within community space, as conducive to crime variables.

Key words authors

Adolescence, Social Maladjustment Process, Community Context, Psychosocial Factors, Conducive to Crime Community Variables.

Key words plus

Social-Comunitary Psychology, Public-Comunity Space, Qualitative Research.
} 


\section{Introducción}

La delincuencia en Chile mantiene las tendencias reportadas en otros países, donde se indica que las mayores cifras de incidencia y prevalencia en conductas antisociales se darían durante la adolescencia y la adultez temprana (Dionne \& Zambrano, 2009). La trasgresión de la ley, según diferentes autores, puede ser comprendida como un epifenómeno de la adolescencia, dado que en este período existe mayor susceptibilidad para la comisión de infracción a la ley (Alarcón, Vinet \& Salvo, 2005; Moffitt, 1993; Musitu et al., 2004). Cabe señalar, sin embargo, que solo una pequeña parte de este grupo continuará delinquiendo más allá de la adolescencia (Frèchette \& Le Blanc, 1987; Le Blanc, 2005; Moffitt, 1993).

Hay coincidencia entre investigadores en señalar que el comportamiento infractor de ley en la población adolescente puede ser un comportamiento aislado o puede constituirse en la expresión de un proceso de desadaptación de larga data que tiene diversas manifestaciones en la vida de los adolescentes (Le Blanc, 2010; Dionne \& Zambrano, 2009). Un aspecto que se ha destacado, es que las trayectorias persistentes de desadaptación social tienen origen durante la infancia y la primera etapa de la adolescencia (Werth, 2006).

Respecto del análisis de los factores asociados a la delincuencia juvenil, se ha utilizado la perspectiva del riesgo psicosocial, la cual permite realizar un análisis de las características personales y del entorno que aumentan la probabilidad de que los jóvenes presenten dificultades en sus trayectorias evolutivas, incorporando la influencia de ciertas variables que, al interactuar entre sí, hacen vulnerables a las personas ante ciertos riesgos. Estas variables son identificadas como factores de riesgo (De la Peña, 2010; Hein, 2002; Sanabria \& Uribe, 2010).

Desde esta perspectiva de análisis, se reconoce también que existirían factores protectores, los cuales tienen un efecto paliativo de las dificultades que pueden estar presentes en los niños o adolescentes y en sus entornos de vida, disminuyendo las probabilidades de incidencia de dificultades en la adaptación y favoreciendo que las personas construyan trayectorias evolutivas resilientes (De la Peña, 2010; Hein, 2002).

El denominado modelo complejo del riesgo, propone la visión del riesgo como proceso y la no linealidad entre causas y efectos. Se asume que no es el riesgo por sí mismo, sino en su relación con las variables protectoras que puede o no generar problemas de ajuste o adaptación (Araujo de Morais, Koller \& Raffaelli, 2010). Las variables protectoras actuarían como antagonistas a la emergencia de problemas que fueron precipitados por los factores de riesgo. Por tanto, la presencia de un evento adverso en la vida del adolescente no es necesariamente una causa en los problemas de ajuste que este pueda presentar. Del mismo modo que no hay linealidad entre causas y efecto, se plantea que son muchas las variables que se pueden interponer a una situación de desventaja, así como de buen ajuste. Los autores de este modelo también proponen que trayectorias de vida diferentes pueden conducir a resultados del desarrollo similares, del mismo modo que trayectorias semejantes no conducen necesariamente a los mismos resultados (Lordelo, 2002).

En diferentes contextos de intervención con población adolescente en dificultades psicosociales, se destaca la relevancia que tienen los factores del contexto de vida en la generación y permanencia de sus problemáticas. Sin embargo, poco se ha precisado respecto de cuáles serían las variables o factores sociocomunitarios que inciden en los procesos de socialización criminógena, asî como los factores sobre los que se debería actuar para mejorar las condiciones de readaptación e integración prosocial de jóvenes que han presentado problemas de ajuste social.

En el contexto chileno, los programas y proyectos orientados a aplicar sanciones penales a los adolescentes infractores de ley, a pesar de atribuir importancia al ámbito comunitario para favorecer procesos de readaptación e integración social (Servicio Nacional de Menores [SENAME], 2007), no cuentan con antecedentes empíricamente validados que permitan focalizar con mayor precisión sobre qué factores específicos habría que actuar 
(Zambrano, Andrade, Pincheira, Fuentealba \& Zambrano, 2011).

En la literatura especializada se señala la existencia de evidencia de que diferentes comportamientos como el uso y abuso de alcohol y drogas, la delincuencia, el crimen y la violencia, se asocian a configuraciones de variados factores de riesgo en distintos niveles de la vida de las personas (De la Peña, 2010; Hein, 2002). Aunque algunos de estos comportamientos pueden ser estacionales y asociados a las características propias de la etapa adolescente, existe también la probabilidad de que bajo ciertas condiciones de riesgo en los ámbitos personal, familiar, escolar y comunitario, se favorezca la generación de estilos relativamente estables de funcionamiento asociados a la desadaptación social (Dionne \& Zambrano, 2009; Meringolo, 2011).

En el ámbito específico de la comunidad se identifican algunos factores de riesgo para la generación de dinámicas de desadaptación social. Sin embargo, hay cierta tendencia a establecer interpretaciones causales entre la pobreza material y este tipo de problemáticas que no facilitan la clara identificación de aquellos factores específicos que puedan tener mayor incidencia, y que por tanto pudiesen constituirse en focos centrales de la acción comunitaria (Dionne \& Zambrano, 2009; Pérez-Luco, Lagos, Rozas \& Santibañez, 2005; Zambrano \& Pérez-Luco, 2004).

Le Blanc (2005) señala la importancia del contexto social en procesos de desadaptación juvenil. Subraya la interacción entre el autocontrol, el control social y las influencias del medio comunitario, concluyendo en sus análisis que existiría una relación compleja entre estos tres aspectos. Factores de la comunidad como la desorganización comunitaria y cultural estarían asociados a una débil aplicación de controles por parte de la misma, lo cual repercutiría en un pobre desarrollo del autocontrol y en una predisposición a responder en menor medida a los controles externos.

Por su parte, Valdenegro (2005) plantea como factores de riesgo a nivel comunitario la baja participación social, el bajo apoyo social percibido y la percepción de ser objeto de prejuicio. Burt (1998), en tanto, sugiere que el abuso de drogas y las conductas de violencia y delincuencia se verían facilitados por ciertas condiciones de la comunidad en la cual el adolescente vive. Este autor señala que la presencia de normas comunitarias favorables a las conductas delictivas, consumo de drogas y adquisición de armas de fuego, así como la influencia de grupos de pares con compromiso delictivo, padres con actitudes favorables hacia el comportamiento problemático, y adicionalmente también falta de cohesión social y organización entre los vecinos, serían características que favorecen un entorno criminógeno.

Adicionalmente, se ha evidenciado el impacto negativo que puede tener la falta de coordinación, continuidad, coherencia e integralidad de la intervención de distintas instituciones en las familias que viven en estos entornos desfavorables (Zambrano et al., 2011). La yuxtaposición de intervenciones sobre las familias, los niños y jóvenes que presentan dificultades, puede favorecer resultados iatrogénicos en la medida en que amplifican la desesperanza, la sensación de falta de control y refuerzan la identidad social negativa.

En suma, en el ámbito comunitario se puede identificar un conjunto de factores de riesgo para la generación de dinámicas de desadaptación social, bajo las cuales la comunidad ofrece oportunidades concretas para la realización de comportamientos desadaptativos, al mismo tiempo que facilita muy poca ayuda para evitarlos.

Canales et al. (2005) plantean la necesidad de abordar la evolución de las trayectorias delictivas desde un enfoque integral con carácter psicosocial, que contemple el contexto socioestructural en el que viven los adolescentes, así como las influencias de la segregación y estratificación social. La prevención del delito, entonces, debería actuar en el espacio local, específicamente en los barrios llamados "vulnerables", los cuales concentrarían factores de riesgo asociados a desventajas sociales y ambientales que favorecen el encapsulamiento territorial del barrio, y terminan generando círculos viciosos de aumento de la violencia, delincuencia y victimización de la población $(\mathrm{Mu}-$ nizaga, 2009). 
Entendiendo que los procesos de desadaptación adolescente son el resultado de la relación compleja de variables de riesgo en diferentes niveles (personal, familiar, escolar y comunitario), se puede destacar que un aspecto relevante en este proceso es el relacional. Si se observan las trayectorias persistentes de desajuste social en los adolescentes, se puede apreciar que en el desarrollo de muchos de ellos, los diferentes recursos de socialización prosocial han fallado, generando importantes vacíos educativos que repercuten en su capacidad para relacionarse y desenvolverse en diferentes entornos. En estas condiciones, se produciría lo que Bonet i Martí (2006) denomina vulnerabilidad relacional, puesto que lo que se encuentra en juego es la calidad de los vínculos de apego, la calidad del clima familiar y social en la escuela, así como la calidad del entorno comunitario para desarrollar los recursos relacionales para la inclusión social.

Los malos tratos, la falta de competencias especializadas en los agentes educativos, la fragmentación de las respuestas institucionales y la falta de una pesquisa y respuesta oportuna, favorecerían esta vulnerabilidad relacional (Le Blanc, Swisher, Vitaro \& Tremblay, 2008; Valverde, 2002).

Considerando los elementos antes expuestos, en la presente investigación se centra el análisis en la calidad de la convivencia barrial, enfatizando los aspectos relacionales que pueden incidir en generar condiciones de riesgo criminógeno favorecedores de trayectorias de desadaptación social. A partir de la caracterización y análisis de la dinámica de un barrio de la ciudad de Temuco que presenta condiciones favorables para la desadaptación social en población infanto-juvenil, se buscó proponer ejes de análisis que permitieran valorar los factores de riesgo, así como los recursos para la prevención comunitaria del comportamiento delictivo en adolescentes. El objetivo del presente estudio es, entonces, delimitar un conjunto de variables del entorno sociocomunitario asociadas a procesos de desadaptación social en general y a dinámicas de infracción de ley en adolescentes en particular, en un barrio de la ciudad de Temuco.

\section{Método}

\section{Participantes}

Los participantes del presente estudio corresponden a diversos actores de la dinámica comunitaria del barrio Vista Verde de la ciudad de Temuco, Chile, dentro de los cuales se pueden diferenciar, por una parte, a líderes y participantes de organizaciones comunitarias y, por otra, a grupos no organizados de jóvenes y niños. Además, se integra en la muestra a diversos agentes técnicos pertenecientes a instituciones, programas y proyectos que realizan actividades en el barrio como: escuela, jardín infantil y sala cuna, consultorio, municipio y algunos programas estatales. En total, la muestra se constituyó con aproximadamente 120 personas.

\section{Técnicas}

Para la producción de los datos se utilizaron diferentes técnicas entre las cuales se pueden identificar: análisis documental, entrevistas, encuestas, talleres diagnósticos, observación participante, análisis de redes sociales, entre otras.

\section{Procedimiento}

La recolección de información responde a la primera etapa del proceso Investigación Acción Participativa (IAP) que, desde el modelo UFRO, comprende de forma general las siguientes etapas: diagnóstico inicial, construcción de la demanda, devolución, problematización y diagnóstico consensuado, formulación del plan estratégico, ejecución del plan de acción y evaluación. Todas estas etapas se caracterizan por someterse a constante retroalimentación y orientarse por un enfoque participativo, que busca la implicación de la mayor cantidad de actores en el proceso.

En concreto, el estudio que aquí se presenta recoge los resultados del análisis correspondiente al diagnóstico consensuado, el cual busca la contrastación analítica y reflexiva de los resultados obtenidos en el diagnóstico inicial con los diversos actores de la comunidad, quienes lo retroali- 
mentan desde sus diversas perspectivas. En este sentido, se sigue una estrategia de construcción de resultados basada en el análisis en progreso, en el cual se privilegian los procesos de interacción dialógica y reflexiva en la construcción del conocimiento. En esta línea, la investigación se sitúa dentro de las perspectivas de investigación dialéctica (Ibáñez, 1993), pues su propósito es colaborar en transformar y democratizar la realidad específica en la cual se realiza un determinado estudio, integrando para esto a las comunidades y al investigador dentro de un mismo sistema que se auto-observa para modificarse.

De este modo, la producción de datos se realizó partiendo de los antecedentes sociodemográficos, para profundizar luego en los aspectos relacionales y representacionales de los diversos actores implicados en la dinámica comunitaria. Estos antecedentes fueron constantemente informados y problematizados/contrastados con los actores locales, con el fin de ahondar en su comprensión, favoreciendo la reflexividad de las personas involucradas y la definición de estrategias, para mejorar aquellos aspectos definidos como problemáticos en el barrio.

\section{Diseño}

Se sigue un diseño de investigación de carácter emergente, ya que permite ir explorando el fenómeno de estudio y modificando el camino y los métodos según sea necesario (Hernández Sampieri, Fernández Collado \& Baptista Lucio, 2010).

\section{Análisis de resultados}

El análisis de los resultados corresponde a un análisis en progreso con carácter emergente, coherente con la metodología utilizada, la cual más que analizar un determinado fenómeno desde categorías analíticas predefinidas, construye, en conjunto con la comunidad, una comprensión consensuada de la realidad, contrastando y depurando progresivamente las dimensiones relevantes respecto del fenómeno.

\section{Resguardos éticos}

Los participantes del estudio (vecinos, profesionales, líderes, niños, jóvenes) accedieron a participar de forma voluntaria y fueron informados acerca de la finalidad del mismo, explicándoles que la información confidencial entregada sería cuidadosamente tratada y que el hecho de participar en la investigación no suponía un riesgo físico, psicológico o social para ellos, todo lo cual se concretó mediante la firma de un consentimiento informado. Adicionalmente, la información producida se fue devolviendo y problematizando con distintos actores del barrio.

\section{Resultados}

Los resultados se exponen describiendo primero aspectos sociodemográficos e históricos del barrio, para analizar posteriormente la dinámica de convivencia barrial, organizada en cinco dimensiones: convivencia comunitaria, acción institucional en la comunidad, norma comunitaria, abordaje familiar del comportamiento infantil y adolescente y apropiación del espacio público.

El Barrio Vista Verde se encuentra en el sector sur-poniente de la ciudad de Temuco y se conformó en los años de 1982 y 1983 a través de la entrega de las viviendas a los habitantes del sector por parte del Servicio de Vivienda y Urbanización (SERVIU). Las familias provenían de diversos sectores de la comuna de Temuco, caracterizados por su condición de vulnerabilidad social. En un primer momento, solo se contaba con las viviendas pero posteriormente se ha ido construyendo equipamiento comunitario y pavimentando las calles del barrio. Actualmente, se emplaza en un sector en donde se han ido ubicando villas de mejor condición social y económica, lo que condiciona su segregación física y social.

Al observar los datos censales del barrio, generados en el año 2002, se evidencia que hay 2.100 habitantes de los cuales 1.014 son hombres y 1.086 son mujeres, que constituyen un aproximado de 500 familias. En cuanto a la distribución por edades, los datos indican que la mayor concentración 
de la población se encuentra entre 10 y 29 años, con $30.4 \%$ del total.

A partir de un análisis global de los resultados, se ha evidenciado que en el barrio existen dificultades en diferentes áreas, que se constituyen en factores de riesgo para la generación de procesos de desadaptación social a partir de interacciones complejas, donde diferentes dimensiones se retroalimentan entre sí, generando contextos de vida riesgosos para los niños, los jóvenes y sus familias. También se han identificado aspectos favorables para el funcionamiento comunitario que se constituyen en potencialidades para resolver los diferentes problemas detectados, según lo muestra la evolución positiva de la comunidad en el último periodo, y tal como también lo confirma la evidencia empírica reportada por la literatura que aborda el tema.

\section{Convivencia comunitaria}

En este ámbito se considera el análisis de la relación entre los diversos actores del barrio, incluyendo las dinámicas de las organizaciones de base.

La Participación/Organización Comunitaria, constituye una subdimensión de esta categoría, entendida como catalizador de la integración social de las personas que viven en el territorio. En el barrio Vista Verde existen variadas organizaciones tanto funcionales como territoriales. Respecto de las primeras, se pueden encontrar clubes de adulto mayor, agrupaciones de mujeres, clubes deportivos, centro general de padres y apoderados de la escuela del sector, comités de ampliación y mejoramiento de vivienda, además de comunidades religiosas católicas y evangélicas. Todas estas organizaciones de uno u otro modo confluyen o interactúan con la Junta de Vecinos del sector y el Consejo Vecinal de Desarrollo, creado al alero del programa "Quiero mi Barrio", del Ministerio de Vivienda y Urbanismo del Gobierno de Chile. A estas dinámicas más o menos formales de participación se deben sumar otras más informales propias de la vida cotidiana en el barrio, dentro de las que se pueden contar: partidos de fútbol organizados por los jóvenes, bazares de ropa y otros artefactos en algunas calles del sector, agrupamiento de jóvenes en algunas esquinas y el juego de los niños en los espacios públicos, parques y plazas, etc.

Respecto a las características de la participación en el barrio, se puede decir que, si bien es cierto que existen dinámicas de compañerismo y solidaridad al interior de las organizaciones, los espacios de participación, y el barrio en general, no están exentos de conflictos entre las personas. Se aprecian pautas relacionales conflictivas tanto al interior de las organizaciones como entre las mismas. A esto se suma que en el sector existirían liderazgos motivados y comprometidos con el barrio, pero que se encuentran sobrecargados por asumir la conducción de las organizaciones sin contar con el apoyo de los demás participantes. Cabe destacar que los espacios de participación existentes, mayoritariamente asociados a organismos interventores externos, reproducen dinámicas clientelistas o semiclientelares en las que la comunidad aparece como receptor más o menos pasivo de los organismos externos y de los procesos que se generan en el barrio, otorgándole a la participación un carácter instrumental, orientado a la obtención de beneficios a corto plazo. Esta dinámica de dependencia y pasividad se repite al interior de la vida del barrio, donde se espera que sean mayoritariamente los líderes quienes resuelvan los problemas.

Estos aspectos le han otorgado significados negativos a la participación y la organización comunitaria en parte de la población, y ha traído como consecuencia un descenso en la implicación de las personas en actividades sociales, transformándose en un círculo vicioso caracterizado por la desconfianza respecto de la participación en relación a sus alcances y logros, inexistencia de recambio y desgaste de los líderes y dirigentes.

Si se observa la progresión temporal de la participación en el barrio, se puede ver que esta ha sido fluctuante a través del tiempo, con niveles altos de implicación en actividades colectivas en años cercanos a la creación del barrio y una disminución conforme pasa el tiempo. Estas variaciones de intensidad se asocian al aumento de los niveles de conflictividad y resolución violenta de conflictos, en el sentido de que a mayor presencia de conflictos, menores niveles de participación. 


\section{Acción institucional en la comunidad}

En esta dimensión se caracteriza la forma en que los agentes técnicos que representan distintos programas, proyectos e instituciones que tienen presencia en el sector, se relacionan con los vecinos y abordan los objetivos de su intervención. Se aprecia que en el barrio Vista Verde funciona una amplia gama de instituciones públicas y privadas de diversa índole y con diferentes grados de inserción y permanencia en la zona. Existen instituciones que se podrían denominar internas y de funcionamiento estable en el barrio, tales como la escuela y el jardín infantil, otras que podrían ser consideradas estables pero externas o con un abordaje sectorial más amplio orientado al control y el orden público, la salud, los programas municipales y distintos programas sociales dependientes del Estado.

Estos agentes técnicos presentan una baja coordinación y trabajo conjunto, frente a lo cual los interventores manifiestan una actitud crítica, considerando la necesidad de establecer nuevos vínculos entre las instituciones, con el fin de asegurar una mejora significativa en las intervenciones requeridas. A pesar de esto, los programas no han logrado solucionar las diversas problemáticas detectadas en el barrio, poseen muy poca claridad respecto de la existencia de los demás y desconocen si la población con la que se encuentran trabajando está siendo abordada por otro programa o institución. Además, la tendencia de los programas que apuntan al desarrollo comunitario ha sido establecer relaciones con un grupo de dirigentes y actores claves del barrio, sin lograr trasmitir más allá de estos su "oferta programática".

Otro aspecto relevante es que la mayoría de los organismos intervienen bajo parámetros establecidos por la institucionalidad, con escasa flexibilidad para atender las particularidades del barrio y tendiendo a responder a los requerimientos de un modo preestablecido. A esto se agrega una corta permanencia de los programas en el barrio, lo que limita la posibilidad de trabajar en torno a procesos $\mathrm{y}$ atender las reales prioridades.

La baja articulación existente es atribuida por los operadores de estos programas a limitaciones de comunicación, desconocimiento de las demás entidades y el trabajo con objetivos y focos distintos, lo cual hace que no se considere relevante la necesidad de una mayor articulación territorial. El barrio Vista Verde resulta en general un foco de trabajo demasiado pequeño que se incluye dentro de un territorio más extenso, lo que invisibiliza sus particularidades.

Un aspecto relevante es que debido a la alta tasa de operadores sociales funcionando en el sector y a la descoordinación entre estos, se produce sobreintervención en los sujetos de atención, favoreciendo desgaste en la población y escaso impacto de las intervenciones en términos de su efectividad, con lo cual con cierta frecuencia se obtienen resultados iatrogénicos. Paralelamente, se han favorecido relaciones de paternalismo y clientelismo, lo que ha limitado el desarrollo de recursos endógenos y ha propiciado relaciones de dependencia.

En suma, la inexistencia de un trabajo articulado, coordinado, sinérgico y centrado en los sujetos de atención a través de la vinculación entre programas y entre estos y los vecinos de la comunidad mediados por procesos participativos, se constituye en un factor obstaculizador del desarrollo de la comunidad y sus miembros, favoreciendo que las dinámicas existentes en el sector se mantengan o se intensifiquen.

\section{Norma comunitaria y comportamiento prosocial}

En esta dimensión se abordan los mecanismos mediante los cuales la comunidad regula su convivencia, describiendo la existencia de reglas, normas y formas de control social formales o informales. En el barrio se observa la existencia de situaciones de inseguridad, caracterizadas principalmente por consumo de alcohol y drogas en el espacio público, actos delictivos, intimidación hacia los vecinos, entre otras. La existencia de este tipo de situaciones actúa como un indicador de la falta de normas comunitarias que favorezcan el comportamiento prosocial o de haber, la débil aplicación de las mismas, principalmente con respecto a las conductas de los jóvenes. Pese a la preocupación explicitada 
por diferentes actores de la comunidad respecto a las situaciones mencionadas, se observa una actitud de pasividad ante lo que ocurre en el barrio basada en la experiencia de intentos frustrados y el miedo a las represalias. En este sentido, hay temáticas que no se abren en ciertos contextos desprotegidos, lo cual impide un tratamiento de los problemas existentes en la comunidad.

Se atribuye la responsabilidad de socializar a los niños de forma prosocial solo a sus familias, desentendiendo la competencia de la comunidad al respecto. Este proceso deja en un estado de anomia a gran parte de los espacios que los niños y adolescentes utilizan fuera del grupo familiar, aportando de este modo en la generación de conductas de riesgo que no son pesquisadas ni sometidas a control social de forma adecuada.

En esta misma línea, si se considera que para las etapas tempranas del desarrollo el grupo de pares constituye un espacio importante de socialización y que las interacciones con dicho grupo no se encuentran adecuadamente supervisadas por lo cual se generan conductas de riesgo en conjunto sin los controles externos necesarios, es más probable que pautas de conducta riesgosas se hagan estables y se perpetúen en el tiempo a través de trayectorias de vida antisociales o desadaptadas.

\section{Apropiación del espacio público}

Si se considera al espacio público como aquel lugar donde ocurre la interacción social de las personas de forma libre y espontánea, principalmente caracterizado por la imposibilidad de imponer controles sociales estructurados y donde la calle parece ser el polo más extremo de lo que puede considerarse público, se puede decir que el barrio Vista Verde cuenta con una cantidad considerable de espacios públicos, entre los que se identifican: una sede comunitaria, una multicancha, espacios de juegos infantiles, cancha de rayuela, a los que se suman áreas verdes, calles y pasajes.

Pese a la generación de actuales modificaciones desde el punto de vista físico-arquitectónico a algunos lugares del barrio, los vecinos del sector manifiestan una percepción negativa respecto del uso de los espacios públicos, principalmente por las noches, los que son descritos como lugares en los que principalmente los jóvenes realizan interacciones mediadas por el consumo de alcohol y drogas, con riñas ocasionales entre ellos. Esto da cuenta de que los mismos lugares pueden ser usados de formas muy diversas, en diferentes momentos y circunstancias, lo cual favorece la existencia de visiones contrapuestas entre los distintos usuarios respecto del uso más idóneo que se le debería dar a un determinado espacio.

En este sentido, se ha evidenciado que la apropiación conflictiva de algunos espacios por grupos de jóvenes acrecienta el sentimiento de inseguridad en los vecinos del sector, disminuyendo su presencia en la vía pública y replegándose hacia espacios más privados como el hogar. Esta conducta de protección deja libres los espacios públicos para ser usados por los grupos con comportamientos disruptivos, con la consecuente reducción de las posibilidades de generar instancias de integración, en las que los diferentes usuarios del entorno sociofísico puedan interactuar de forma diversificada y generar las estrategias de control social necesarias para hacer un uso no conflictivo de los diferentes espacios.

Así mismo, se ha evidenciado que entre los vecinos prima una identidad social de barrio asociada a la marginalidad y la delincuencia, aspecto reforzado por los medios de comunicación y habitantes de la ciudad en general, a través de una percepción negativa respecto de Vista Verde. De este modo, la apropiación conflictiva de los espacios públicos y su uso para actividades ilícitas, transfiere contenidos simbólicos negativos al entorno barrial, lo afecta la identidad del sector en general, con los consecuentes impactos en las identidades individuales de sus habitantes. Esta dinámica, a su vez, tiene repercusiones importantes en el apego que las personas sienten hacia su barrio, el cual tendería a ser bastante ambivalente y que se refleja en sentimientos de afecto mezclados con deseos de no pertenencia.

A los jóvenes y niños esta dinámica los afecta de forma directa, pues corresponden a unos de los principales usuarios del entorno sociofísico del barrio, además de que se encuentran en el proceso crítico de construcción y consolidación de su identidad. Es 
en este sentido que las dinámicas de apropiación del espacio público y la identidad barrial suponen variables críticas respecto de la generación de procesos de desadaptación social.

\section{Abordaje familiar del comportamiento infantil y adolescente}

Los relatos de los vecinos del barrio están cargados por historias de vida difíciles, desde la infancia, tanto a nivel personal como del grupo familiar, donde se mencionan por un lado historias de esfuerzo y sacrificio, al mismo tiempo que se naturalizan temas como la violencia intrafamiliar, el abuso de alcohol de las figuras parentales, las pautas de crianza sexistas e incluso, en alguna ocasión, la presencia de hermanos mayores, tíos o hijos que cumplen condena en centros penitenciarios.

Se destaca el escaso, insuficiente o inadecuado control parental presente en algunas familias que da paso a la existencia de tiempo libre sin supervisión, por lo que la influencia del grupo de pares no está adecuadamente controlada; el ejercido del cuidado de los niños es generalmente monoparental, y se evidencia parentalización de los niños y niñas quienes quedan al cuidado de sus hermanos menores o de otros niños del barrio. Los vecinos y técnicos del sector reconocen la existencia de múltiples factores de riesgo a nivel familiar, señalando que algunas familias presentan diversas dificultades relacionales, carencias en el área económica, dificultades de salud mental, entre otras, todas cuestiones que afectarían la socialización de los niños y adolescentes. Todo esto propiciaría pautas de comportamiento riesgoso por parte de los integrantes más jóvenes de las familias.

\section{Discusión}

Los resultados muestran que el barrio Villa Vista Verde, a pesar de sus particularidades, presenta un perfil que tipifica de un modo general a barrios que favorecen condiciones para propiciar procesos de desadaptación social en niños y adolescentes (Azocar et al., 2009; Burt, 1998; Hein, 2002; Le Blanc, 2005; Valdenegro, 2005). Las categorías que emergen en el estudio como dimensiones sociocomunitarias críticas en procesos de desadaptación adolescentes, dan cuenta de variables asociadas a la calidad de la convivencia comunitaria (Arango, 2007). Todas estas dimensiones reportan importantes debilidades en la calidad del entorno relacional en el que se desenvuelven y socializan los niños y jóvenes del sector. Esto implica, para el desarrollo de los adolescentes particularmente para aquellos que tienen dificultades familiares, un obstáculo para el aprendizaje de comportamientos que se ajusten a las normas y al respeto por los otros, así como cierta disposición a comportamientos de riesgo, los que se ven facilitados por los escasos mecanismos de supervisión y control social. Tal como lo refiere Bonet (2006) las condiciones barriales criminógenas favorecerían la vulnerabilidad relacional.

Esto es coincidente con lo propuesto por Le Blanc $(2005,2010)$, a partir de la teoría de la regulación social. Este autor señala que el autocontrol se va configurando en las personas a través de la interacción con figuras de apego, quienes serían los responsables de plantear las restricciones y propiciar el modelaje conductual. Bajos niveles de autocontrol, asociados a una baja capacidad de responder al control social (mediado por el contexto familiar), en un medio donde existen oportunidades de generar conductas desviadas, propiciaría la aparición de estilos de conducta delictiva o problemática y, si se dan las condiciones, trayectorias evolutivas asociadas a la criminalidad.

Se podría concluir que el entorno comunitario del barrio estudiado presenta un conjunto de características que permiten hablar de vulnerabilidad social, en el sentido de que presenta procesos sociales que ponen en riesgo el desarrollo saludable de una proporción relevante de sus habitantes. Las diferentes variables detectadas se retroalimentan mutuamente para la generación de un contexto altamente propicio para la generación de dinámicas psicosociales problemáticas, lo cual coincide con lo expuesto por Hein (2002) respecto de la complejidad e interrelación de los diferentes niveles de riesgo.

Lo anterior no significa que no se presenten a la par factores protectores, los que se constituirían en 
la principal palanca para el cambio de las actuales condiciones de vida barrial. Entre estos factores destacan cierto nivel de organización y participación, motivación de parte de algunos líderes (a pesar de su sobrecarga) por aportar al desarrollo de la comunidad y la validación de la escuela en la comunidad, espacio que permitiría junto al jardín infantil potenciar espacios de encuentro y formación para los padres en cuestiones relativas a la crianza de los hijos. A pesar de su fragmentación, hay un conjunto de recursos interinstitucionales trabajando en el barrio que, con mayor coordinación y redefinición del abordaje de la intervención, se constituyen en un potencial para la dinámica barrial. En esta línea, es relevante favorecer procesos de fortalecimiento comunitario que permitan incidir de un modo positivo en las variables de riesgo, mediante el desarrollo de acciones acordes con los ejes identificados como claves para esto.

En síntesis, se puede afirmar que las variables delimitadas en el estudio aportan por una parte a la comprensión del fenómeno desde un punto de vista más integral y complejo, al mismo tiempo que proveen de claves para la generación de un abordaje de los ámbitos considerados críticos en cuanto favorecedores de procesos desadaptativos. En el caso de este barrio específico, es imprescindible una intervención comunitaria que incida en múltiples niveles de la vida barrial en el mediano y largo plazo. Los focos críticos por abordar se relacionan con las cinco dimensiones surgidas en este estudio, debiéndose orientar la acción comunitaria a: potenciar la convivencia barrial positiva, fortalecer las normas comunitarias y los mecanismos de regulación de la vida barrial, propiciar recursos familiares que favorezcan la parentalidad positiva, propiciar la gestión asociada de la oferta institucional con las organizaciones barriales y posibilitar una apropiación positiva de los espacios públicos.

Como proyección y desafío de este estudio, se deriva la necesidad de contrastar estos resultados preliminares con los obtenidos en otros barrios, con el fin de evaluar la pertinencia del perfil sociocomunitario de los factores de riesgo criminógenos. Los datos producidos aquí, por tratarse de una investigación situada con fines de intervención, tienen la restricción de ser solo válidos para el caso en estudio. Pese a ello, la coincidencia de los resultados con los reportados en la literatura que aborda el tema, permiten suponer que pueden ser de utilidad para indagar con mayor precisión la dinámica barrial en contextos que aparecen de mayor riesgo y que requieren de estrategias efectivas de intervención. En esta perspectiva, las dimensiones propuestas, miradas en un continuo entre la protección y el riesgo, pueden ser consideradas en la construcción de un instrumento que permita detectar recursos comunitarios para favorecer la convivencia e integración social, facilitando así la identificación más rigurosa de los focos necesarios de abordar en una estrategia comunitaria.

\section{Referencias}

Alarcón, P., Vinet, E. \& Salvo, S. (2005). Estilos de personalidad y desadaptación social durante la adolescencia. Psykhe, 14(1), 3-16.

Arango, C. (2007). Psicología comunitaria de la convivencia. Cali, Co: Programa Editorial Universidad del Valle.

Araujo de Morais, N., Koller, S. H. \& Raffaelli, M. (2010). Eventos estressores e indicadores de ajustamento entre adolescentes em situação de vulnerabilidade social no Brasil. Universitas Psychologica, 9(3), 787-806.

Azocar, B., Dorvillius, E., Echevarria, P., Filliaudeau, H., Jamoulle, P., Joubert, M., et al. (2009). Elementos generales para entender la generación de conductas de riesgo en sectores vulnerables [Les conduites à risques. Penser et agir la prévention] (S. Serrano, Trad.). El Observador, 5, 120-167.

Bonet i Martí, J. (2006). La vulnerabilidad relacional: análisis del fenómeno y pautas de intervención. Redes. Revista Hispana para el Análisis de Redes Sociales, 11(4), 1-17. Disponible en http://revistaredes.rediris.es

Burt, M. R. (1998). ¿Por qué debemos invertir en el adolescente? Documento preparado para la conferencia Salud Integral de los Adolescentes y Jóvenes de América Latina y el Caribe (1996, julio), OPSOMS, Washington, DC. 
Canales, M., Fuentealba, T., Jiménez, J., Morales, J., Cottet, P. \& Agurto, I. (2005). Factores que inciden en la comisión de delitos graves en adolescentes infractores de ley. El Observador, 1, 49-72.

De la Peña, M. E. (2010). Conducta antisocial en adolescentes: factores de riesgo y protección. Tesis Doctoral, Universidad Complutense de Madrid, España.

Dionne, J. \& Zambrano, A. (2009). Intervención con adolescentes infractores de ley. El Observador, 5, 35-56.

Fréchette, M. \& Le Blanc, M. (1987). Délinquances et délinquants. Chicoutimi: Gaëtan Morin.

Hein, A. (2002). Factores de riesgo y delincuencia juvenil: revisión de la literatura nacional e internacional. Santiago de Chile: Paz Ciudadana.

Hernández Sampieri, R., Fernández Collado, C. \& Baptista Lucio, P. (2010). Metodología de la investigación. México: McGraw-Hill.

Ibáñez, J. (1993). El análisis de la realidad social. Métodos y técnicas de investigación. Madrid: Alianza Universidad Textos.

Le Blanc, L., Swisher, R., Vitaro, F. \& Tremblay, R. (2008). Journal of High school social climate and antisocial behavior: A 10 year longitudinal and multilevel study. Journal of Research on Adolescence, 18(3), 395-419.

Le Blanc, M. (2005). Self-control and social control of deviant behavior in context: Development and interactions along the life course (A. Emaldía, Trad.). En P. -O. Wikstrom \& R. Sampson (Eds.), The social contexts of pathways in crime: Development, context, and mechanisms (pp. 1-55). Cambridge: Cambridge University Press.

Le Blanc, M. (2010). Un paradigme développemental pour la criminologie : développement et autorégulation de la conduite déviante. Criminologie, 43(2), 401-428.

Lordelo, E. R. (2002). Contexto e desenvolvimento humano: Quadro conceitual. En E. Lordelo, A. M. Carvalho \& S. Koller (Eds.), Infância brasileira e contextos de desenvolvimento (pp. 5-18). Sao Paulo/ Salvador: Casa do Psicólogo/EDUFBA.
Meringolo, P. (2011). Juvenile justice system in Italy. Researches and interventions. Trabajo no públicado, University of Florence, Departament of Psychology.

Moffitt, T. (1993). Adolescence-limited and life-coursepersistent antisocial behavior: A developmental taxonomy. Psychological Review, 100(4), 674-701.

Munizaga, A. M. (2009). Consideraciones relevantes para la prevención del delito en barrios vulnerables. Fundación Paz Ciudadana, 8, 1-12.

Musitu, G., Buelga, S., Lila, M. \& Cava, M. (2004). Familia y adolescencia. Madrid: Síntesis.

Pérez-Luco, R., Lagos, L., Rozas, R. \& Santibañez, J. (2005). Impacto vital del paso por un COD en la adolescencia. La experiencia de cuatro adultos de Temuco. Revista de Psicología de la Universidad de Chile, 14(2), 27-46.

Sanabria, A. M. \& Uribe, A. F. (2010). Factores psicosociales de riesgo asociados a conductas problemáticas en jóvenes infractores y no infractores. Diversitas. Perspectivas en Psicología, 6(2), 257-274.

Servicio Nacional de Menores. (2007). Sistema nacional de atención socioeducativa para adolescentes infractores de ley. Periodo 2006-2007. Santiago de Chile: Autor.

Valdenegro, B. A. (2005). Factores psicosociales asociados a la delincuencia juvenil. Psykhe, 14(2), 33-42.

Valverde, J. (2002). El diálogo terapéutico en exclusión social: aspectos educativos y clínicos. España: Narcea.

Werth, F. (2006). Prevención social del delito: pautas para una intervención temprana en niños y jóvenes. Santiago de Chile: Fundación Paz Ciudadana.

Zambrano, A., Andrade, C., Pincheira, C., Fuentealba, J. \& Zambrano, V. (2011). Variables asociadas a la readaptación e integración social de adolescentes infractores de ley (Informe de proceso). Temuco, Chile: Proyecto FONDEF D08I1205.

Zambrano, A. \& Pérez-Luco, R. (2004). Construcción de identidad en jóvenes infractores de ley, una mirada desde la Psicología Cultural. Revista de Psicología de la Universidad de Chile, 13(1), 115-132. 
